\title{
Duty-cycle and phase spacing error correction circuit for high-speed serial link
}

\author{
Hyochang Kim ${ }^{1,2}$, Ook Kim ${ }^{2}$, and Changsik Yoo ${ }^{1 \text { a) }}$ \\ ${ }^{1}$ Department of Electronnics Engineering, Hanyang University, \\ 222, Wangsimni-ro, Seoungdong-gu, Seoul 131-791, Korea \\ 2 AlphaSolutions Inc., \\ 344, Pangyo-ro, Bundang-gu, Seongnam-si, Gyeonggi-do, Korea \\ a)csyoo@hanyang.ac.kr
}

\begin{abstract}
Duty cycle and phase spacing of multi-phase clock are converted to an analog voltage by low-pass filtering a clock pulse and quantized by a low-power analog-to-digital converter (ADC). The pull-up and pull-down strengths and the delay of clock buffer are controlled till the duty cycle and phase spacing measured by the ADC become equal to desired values. A prototype has been implemented in a $28-\mathrm{nm}$ CMOS process for a $12-\mathrm{Gbps}$ serial link transceiver and occupies only $0.0014-\mathrm{mm}^{2}$. Experimental results show the deterministic jitter decreases from 8.12-ps to 0.91-ps by the proposed duty cycle and phase spacing error correction technique. While operating with a $1.0-\mathrm{V}$ supply, the additional power consumed for the duty cycle and phase spacing error correction is only $76-\mu \mathrm{W}$.
\end{abstract}

Keywords: duty-cycle, phase spacing, analogue-to-digital converter (ADC), CMOS, deterministic jitter

Classification: Integrated circuits

\section{References}

[1] C. Hou: “A smart design paradigm for smart chips," ISSCC Dig. Tech. Papers (2017) 8 (DOI: 10.1109/ISSCC.2017.7870240).

[2] B. Casper and F. O'Mahony: "Clocking analysis, implementation and measurement techniques for high-speed data links-a tutorial," IEEE J. SolidState Circuits 56 (2009) 17 (DOI: 10.1109/TCSI.2008.931647).

[3] Y.-H. Song, et al.: “A $0.47-0.66 \mathrm{pJ} / \mathrm{bit}, 4.8-8 \mathrm{~Gb} / \mathrm{s} \mathrm{I} / \mathrm{O}$ transceiver in $65 \mathrm{~nm}$ CMOS,” IEEE J. Solid-State Circuits 48 (2013) 1276 (DOI: 10.1109/JSSC. 2013.2249812).

[4] S. Yuan, et al.: “A $70 \mathrm{~mW} 25 \mathrm{~Gb} / \mathrm{s}$ quarter-rate SerDes transmitter and receiver chipset with $40 \mathrm{~dB}$ of equalization in $65 \mathrm{~nm}$ CMOS technology," IEEE Trans. Circuits Syst. I, Reg. Papers 63 (2016) 939 (DOI: 10.1109/TCSI.2016. 2555250).

[5] C. Yoo, et al.: "Open-loop full-digital duty cycle correction circuit," Electron. Lett. 41 (2005) 635 (DOI: 10.1049/el:20050776).

[6] C. Park, et al.: "A 1.8-GHz self-calibrated phase-locked loop with precise I/Q matching,” IEEE J. Solid-State Circuits 36 (2001) 777 (DOI: 10.1109/ 
4.918915).

[7] M. Kossel, et al: "A T-Coil-Enhanced $8.5 \mathrm{~Gb} / \mathrm{s}$ high-swing SST transmitter in $65 \mathrm{~nm}$ bulk CMOS with $<-16 \mathrm{~dB}$ return loss over $10 \mathrm{GHz}$ bandwidth," IEEE J. Solid-State Circuits 43 (2008) 2905 (DOI: 10.1109/JSSC.2008.2006230).

[8] C. Menolfi, et al: "A $28 \mathrm{~Gb} / \mathrm{s}$ source-series terminated TX in $32 \mathrm{~nm}$ CMOS SOI,” ISSCC Dig. Tech. Papers (2012) 334 (DOI: 10.1109/ISSCC.2012. 6177035).

[9] L. Xia, et al.: "Sub-2-ps, static phase error calibration technique incorporating measurement uncertainty cancellation for multi-Gigahertz time-interleaved T/H circuits," IEEE Trans. Circuits Syst. I, Reg. Papers 59 (2012) 276 (DOI: 10.1109/TCSI.2011.2162382).

[10] J. Doernberg, et al.: "Full-speed testing of A/D converters," IEEE J. SolidState Circuits 19 (1984) 820 (DOI: 10.1109/JSSC.1984.1052232).

[11] M. Mota and J. Christiansen: "A high-resolution time interpolator based on a delay locked loop and an RC delay line," IEEE J. Solid-State Circuits 34 (1999) 1360 (DOI: 10.1109/4.792603).

[12] L. M. Lee, et al.: "A sub-10-ps multiphase sampling system using redundancy," IEEE J. Solid-State Circuits 41 (2006) 265 (DOI: 10.1109/ JSSC.2005.859883).

[13] Y.-H. Song, et al.: "An $8-16 \mathrm{~Gb} / \mathrm{s}, 0.65-1.05 \mathrm{pJ} / \mathrm{b}$ voltage-mode transmitter with analog impedance modulation equalization and sub-3 ns power-state transitioning," IEEE J. Solid-State Circuits 49 (2014) 2631 (DOI: 10.1109/ JSSC.2014.2353795).

[14] J. Kim, et al:: "A 16-to- $40 \mathrm{~Gb} / \mathrm{s}$ quarter-rate NRZ/PAM4 dual-mode transmitter in $14 \mathrm{~nm}$ CMOS," ISSCC Dig. Tech. Papers (2015) 60 (DOI: 10. 1109/ISSCC.2015.7062925).

[15] T. C. Hsueh, et al.: "A $25.6 \mathrm{~Gb} / \mathrm{s}$ differential and DDR4/GDDR5 dual-mode transmitter with digital clock calibration in $22 \mathrm{~nm}$ CMOS," ISSCC Dig. Tech. Papers (2014) 444 (DOI: 10.1109/ISSCC.2014.6757506).

[16] W. Dettloff, et al.: "A $32 \mathrm{~mW} 7.4 \mathrm{~Gb} / \mathrm{s}$ protocol-agile source-series-terminated transmitter in $45 \mathrm{~nm}$ CMOS SOI," ISSCC Dig. Tech. Papers (2010) 370 (DOI: 10.1109/ISSCC.2010.5433825).

\section{Introduction}

In a scaled CMOS process, accurate delivery of a high-frequency clock signal with low power is very challenging due to the substantial increase of metal resistance and fringing capacitance together with lowered supply voltage $[1,2]$. As a solution for this, high-speed serial link transceiver usually employs multi-phase clocking which allows the use of a lower frequency clock for a given data rate [3, 4]. The random mismatch, however, causes duty cycle error and unequal phase spacing of multi-phase clock which appear as deterministic jitter (DJ) degrading the performance of high-speed serial link $[5,6,7,8]$. Duty cycle and phase spacing error of multi-phase clock can be detected by measuring clock pulse width. The detected errors are corrected by controlling the pulse width and/or delay. The accuracy of the error detection and thus the error correction can be improved by asynchronous sampling which increases the effective sampling rate $[9,10,11,12]$. Because the frequency of an asynchronous sampling clock is different from that of a system clock, there must be an additional clock generation circuit $[13,14,15,16]$. 
This Letter describes a simple detection and correction schemes for duty cycle and phase spacing error for a 12-Gbps serial link transceiver. A low-power analogue-to-digital converter (ADC) detects duty cycle and phase spacing error which is corrected by controlling the pull-up and pull-down strengths and the delay of clock buffer.

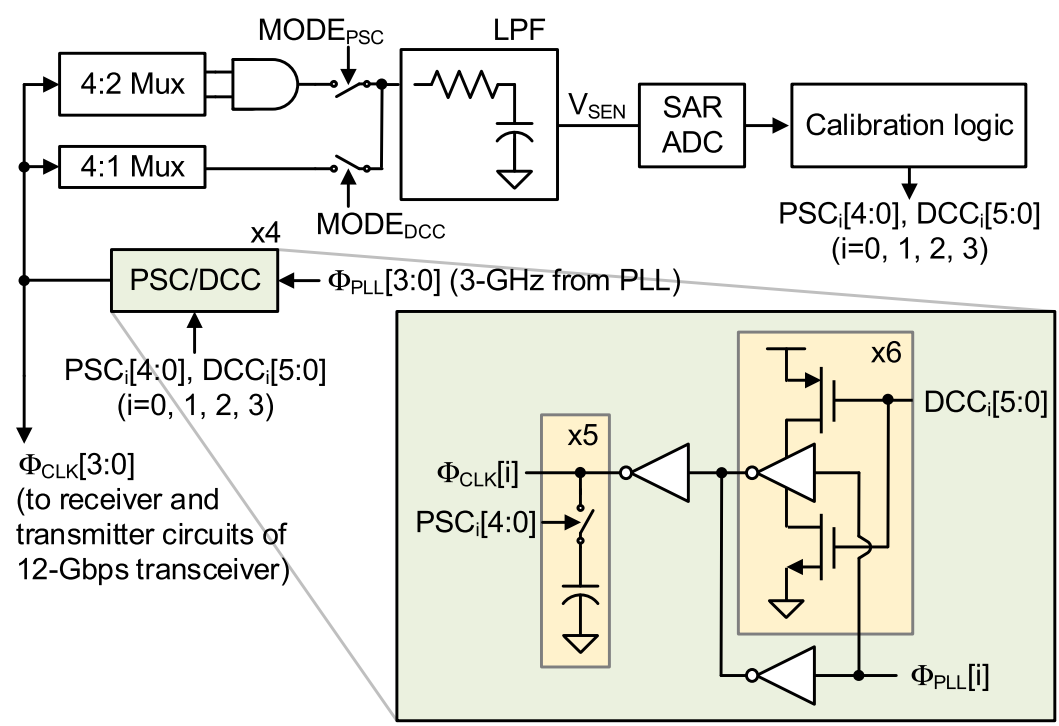

Fig. 1. Architecture of the proposed duty cycle and phase spacing error correction circuit (DPECC).

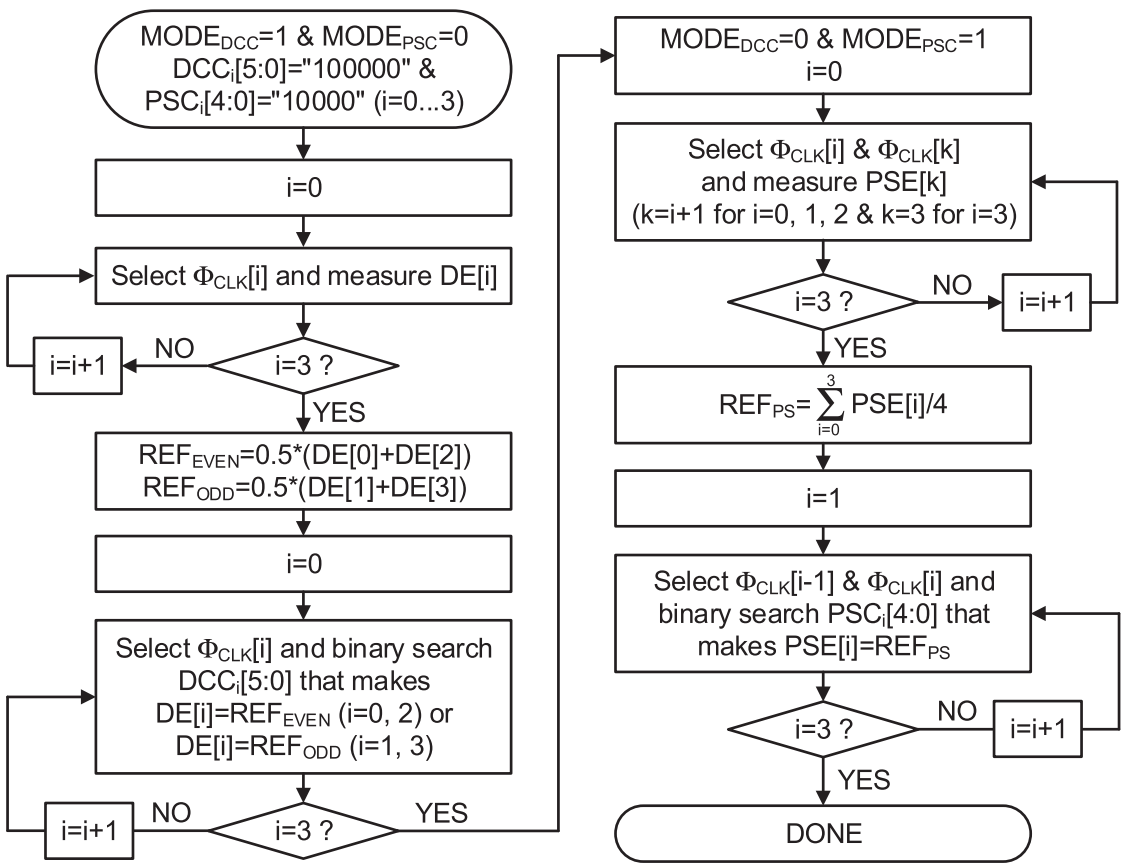

Fig. 2. Flow chart of the proposed DPECC operation.

\section{Architecture and implementation}

Fig. 1 shows the architecture of the proposed duty cycle and phase spacing error correction circuit (DPECC) for a 12-Gbps serial link transceiver. To lower the power required to deliver clock signal, four-phase 3-GHz clock is used for 12-Gbps 
operation. An on-chip phase locked loop (PLL) provides the four-phase 3-GHz clock $\Phi_{P L L}[0: 3]$ which is applied to the phase spacing corrector (PSC) and duty cycle corrector (DCC). The 4:1 multiplexer selects a clock and the 4:2 multiplexer selects two adjacent-phase clocks from the PSC/DCC output $\Phi_{C L K}[0: 3]$, respectively for duty cycle correction and phase spacing error correction. The calibration logic is implemented in an embedded micro-controller unit (MCU) with a desired software engine.

The flow chart of the DPECC operation is show in Fig. 2. The DPECC performs the duty cycle correction first and then corrects the phase spacing error with duty corrected clock. During the duty cycle correction mode $\left(M O D E_{D C C}=\right.$ "1"), the output of the 4:1 multiplexer is low-pass filtered to get $V_{S E N}$ which is quantized by a 9-bit successive approximation register (SAR) ADC. The voltage level of $V_{S E N}$ is proportional to the duty cycle of the selected clock and therefore the SAR ADC output indicates the duty cycle. The duty cycles of $\Phi_{C L K}[0: 3]$ are all measured and the ADC outputs are stored in the calibration logic. Let's denote the ADC output for $\Phi_{C L K}[i]$ indicating its duty cycle as $D E[i]$. The clock $\Phi_{C L K}[2]$ is complementary (inverted) one of the clock $\Phi_{C L K}[0]$. Therefore, the average duty cycle of $\Phi_{C L K}[0]$ and $\Phi_{C L K}[2]$ is 50-\% even with duty cycle error. The same is true for $\Phi_{C L K}[1]$ and $\Phi_{C L K}[3]$ as well. Exploiting this, the average of $D E[0]$ and $D E[2]$ is used as the reference code $R E F_{E V E N}$ to correct the duty cycles of $\Phi_{C L K}[0]$ and $\Phi_{C L K}[2]$. The average of $D E[1]$ and $D E[3]$ is used as the reference code $R E F_{O D D}$ to correct the duty cycles of $\Phi_{C L K}[1]$ and $\Phi_{C L K}[3]$. After the reference codes $R E F_{E V E N}$ and $R E F_{O D D}$ are obtained, the calibration logic moves to the next step, that is, duty cycle correction by controlling the codes $D C C_{i}[5: 0]$ for clock $\Phi_{C L K}[i]$ where $i=0,1,2,3$. By varying the pull-up and pull-down strengths by $D C C_{i}[5: 0]$, the duty cycle of $\Phi_{C L K}[i]$ can be corrected. Until the ADC output $D E[i]$ becomes equal to $R E F_{E V E N}$ (for $i=0,2$ ) or $R E F_{O D D}$ (for $i=1,3$ ), the duty cycle correction code $D C C_{i}[5: 0]$ is found by binary search.

When the duty cycles of $\Phi_{C L K}[0: 3]$ are all corrected to be $50-\%$, the DPECC moves to the phase spacing error correction mode $\left(M O D E_{P S C}=\right.$ "1"). The 4:2 multiplexer selects two adjacent-phase clocks $\Phi_{C L K}[i]$ and $\Phi_{C L K}[i+1]$ which are then applied to the AND gate. The pulse width of the AND gate output is equal to the phase spacing between $\Phi_{C L K}[i]$ and $\Phi_{C L K}[i+1]$ and therefore the voltage level of the low-pass filtered $V_{S E N}$ is proportional to their phase spacing. Let's denote the ADC output as $P S E[i+1]$ when $\Phi_{C L K}[i]$ and $\Phi_{C L K}[i+1]$ are selected. Even with phase spacing error, $\sum_{i=0}^{3} P S E[i]$ indicates one clock period and its quarter $\sum_{i=0}^{3} P S E[i] / 4$ is used as the reference code $R E F_{P S}$ for the phase spacing correction. After the reference code $R E F_{P S}$ is obtained, the phase spacing error is corrected by controlling the delay of each clock with the delay control code $P S C_{i}[4: 0]$. Until the ADC output $P S E[i]$ becomes equal to $R E F_{P S}$, the phase spacing correction code $P S C_{i}[4: 0]$ is found by binary search for $i=1,2,3$. We don't need to control the delay of the clock $\Phi_{C L K}[0]$ because $P S E[0]$ would be $R E F_{P S}$ if $P S E$ [1], PSE[2], and PSE[3] are all equal to $R E F_{P S}$. 


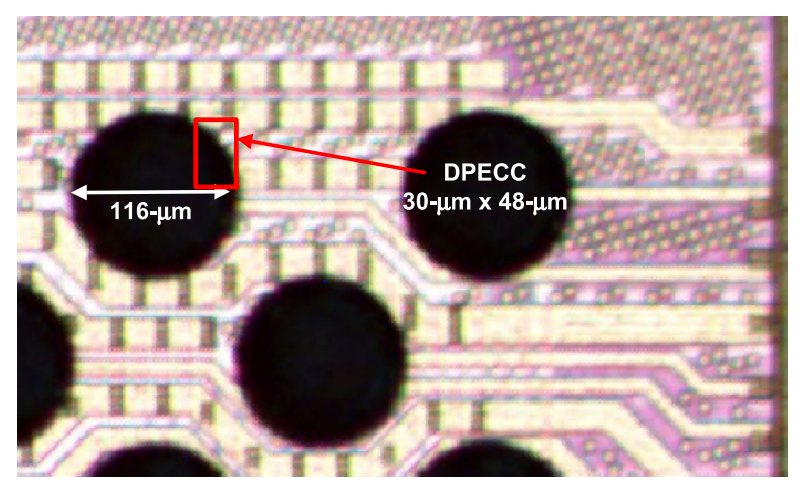

(a)

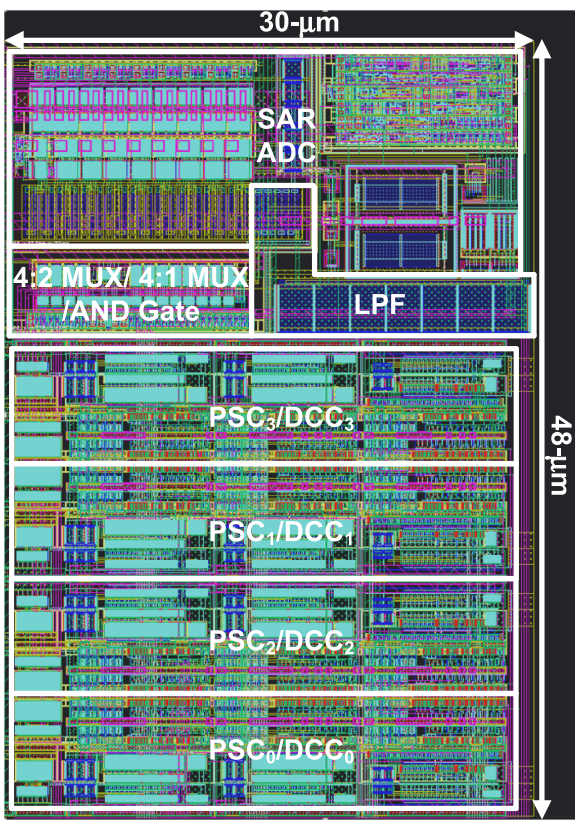

(b)

Fig. 3. Chip microphotograph (a) and its layout (b).

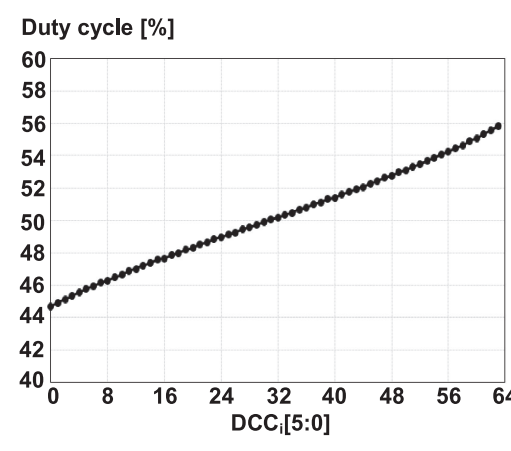

(a)

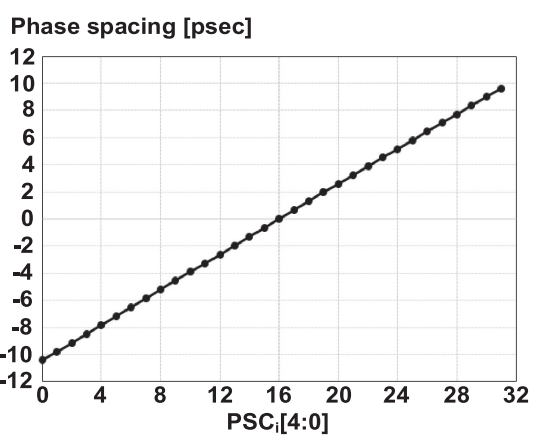

(b)

Fig. 4. Simulation result of duty-cycle correction range (a) and phase spacing error correction range (b).

\section{Experimental and simulation results}

In order to verify the operation of the proposed DPECC, a 12-Gbps transmitter has been implemented in a $28-\mathrm{nm}$ CMOS process. The chip microphotograph and its layout are shown in Fig. 3 and the active area of the DPECC is $0.0014-\mathrm{mm}^{2}$. The 


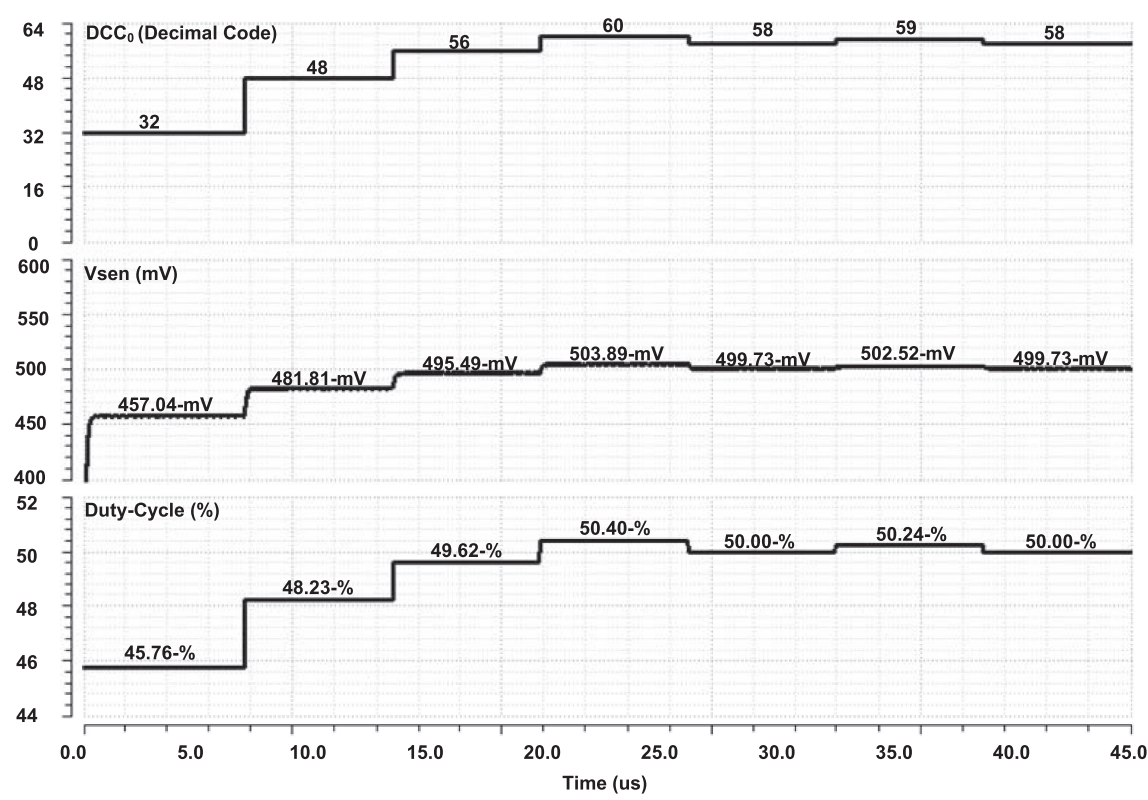

Fig. 5. Simulated transient response of the duty cycle correction when the duty cycle is initially $45.76-\%$.
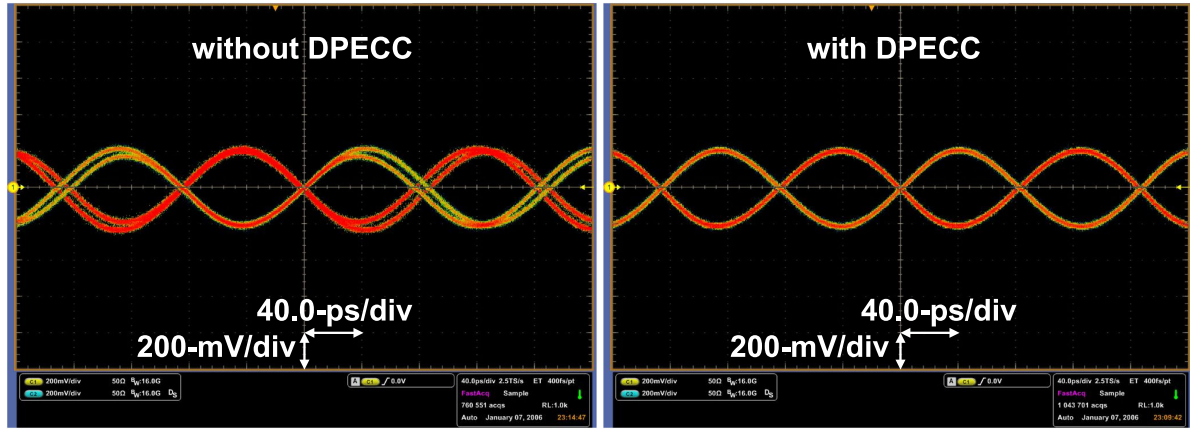

(a)
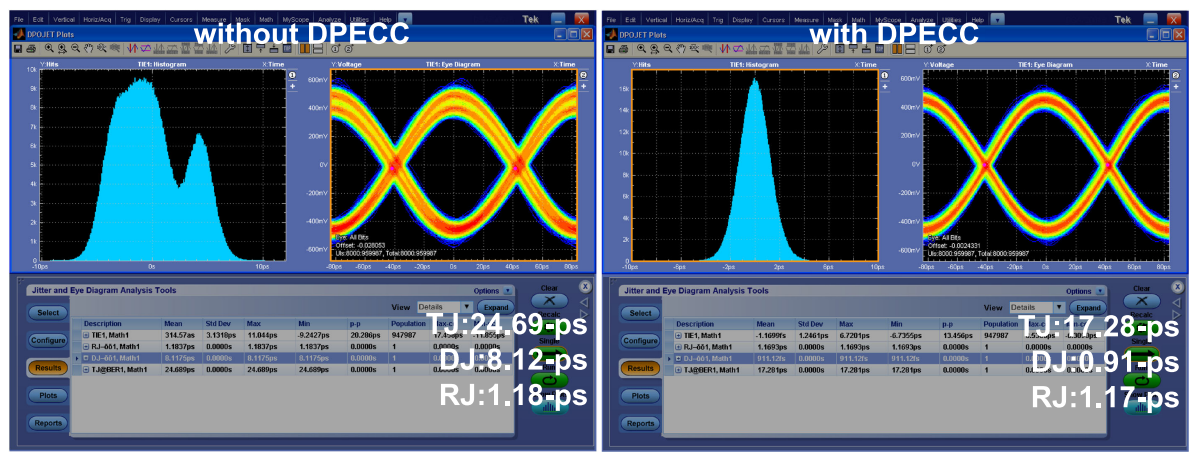

(b)

Fig. 6. Measured results for 12-Gbps clock pattern. (a) Output waveform and (b) Eye diagram and jitter histogram.

transmitter consists of a serializer clocked by a four-phase $3-\mathrm{GHz}$ clock and a differential output driver which is terminated by differential $100-\Omega$ at both source and sink ends. Operating with a $1.0-\mathrm{V}$ supply, the additional power consumed for the DPECC operation is $76-\mu \mathrm{W}$. 
The simulated correction ranges of duty cycle and phase spacing error are plotted versus the control codes $D C C_{i}[5: 0]$ and $P S C_{i}$ [4:0], respectively in Fig. 4. The duty cycle is correctable from $44.7-\%$ to $55.8-\%$ with the resolution of $0.28-\%$ and the phase spacing error is correctable from -10.4 -ps $\left(-11.2^{\circ}\right)$ to 9.6 -ps $\left(10.4^{\circ}\right)$ with the resolution of 0.65 -ps $\left(0.7^{\circ}\right)$. The bandwidth of the low-pass filter (LPF) generating $V_{S E N}$ is designed to be $3-\mathrm{MHz}$ which is low enough to remove the ripple of 3-GHz clock. The LPF consisting of $100-\mathrm{k} \Omega \mathrm{p}+$ polysilicon resistor and $710 \mathrm{-fF}$ n-channel MOS capacitor has 2.2-MHz bandwidth. The 9-bit SAR ADC with $185-\mathrm{kS} / \mathrm{s}$ is designed so its full-scale range and quantization resolution do not to limit the accuracy of the duty cycle and phase spacing error correction. Fig. 5 show the simulated transient response of $V_{S E N}$ and duty-cycle according to control code $D C C_{0}[5: 0]$ by using binary search when the duty cycle of $\Phi_{P L L}[0]$ is $46-\%$ to verify DPECC algorithm. In this case, $R E F_{E V E N}$ code is 255 and equivalent voltage level is $0.5-\mathrm{V}$.

To see the effect of the proposed DPECC, a 12-Gbps clock pattern is driven to the output. The measured waveform and jitter histogram are shown in Fig. 6a and Fig. $6 \mathrm{~b}$, respectively without and with the DPECC. Fig. $6 \mathrm{a}$ show single $+/-$ signals to check transient signal and Fig. 6b show differential signal eye diagram and jitter histogram by taking rising and falling edge to check 1010 and 0101 pattern at the same time. When the DPECC is disabled, the jitter histogram shows multiple peaks resulting from duty cycle and phase spacing error. With the DPECC enabled, the jitter histogram gathers to have one peak and the DJ decreases from 8.12-ps to 0.91-ps. The total jitter (TJ) decreases from 24.69-ps to 17.28-ps while the random jitter $(\mathrm{RJ})$ is negligibly affected by the DPECC.

\section{Conclusion}

In this Letter, a duty cycle and phase spacing error correction circuit is presented for a high-speed serial link transceiver. The duty cycle and phase spacing of multiphase clock are converted to an analogue voltage by low-pass filtering a clock pulse and quantized by a low-power SAR ADC to obtain the reference codes for the error correction. Then, the pull-up and pull-down strengths and the delay of clock buffer are varied till the measured duty cycle and phase spacing become equal to the reference codes. A prototype circuit for a 12-Gbps serial link transceiver has been implemented in a $28-\mathrm{nm}$ CMOS process and occupies $0.0014-\mathrm{mm}^{2}$. With the propose duty cycle and phase spacing error correction circuit, the DJ decreases from 8.12-ps to 0.91-ps.

\section{Acknowledgments}

A part of this work was supported by the National Research Foundation of Korea (NRF) grant funded by the Korea government (MSIP) (NRF-2016R1D1A1 B03930310). 